\title{
Pressure Induced Compression of Flatbands in Twisted Bilayer Graphene
}

\author{
Bheema Lingam Chittari, ${ }^{1}$ Nicolas Leconte, ${ }^{1}$ Srivani Javvaji, ${ }^{1}$ and Jeil Jung ${ }^{1}$ \\ ${ }^{1}$ Department of Physics, University of Seoul, Seoul 02504, Korea
}

\begin{abstract}
We investigate the bandwidth compression due to out of plane pressure of the moire flatbands near charge neutrality in twisted bilayer graphene for a continuous range of small rotation angles of up to $\sim 2.5^{\circ}$. The flatband bandwidth minima angles are found to grow linearly with interlayer coupling $\omega$ and decrease with Fermi velocity. Application of moderate pressure values of up to $2.5 \mathrm{GPa}$ achievable through a hydraulic press should allow to access a flatband for angles as large as $\sim 1.5^{\circ}$ instead of $\sim 1^{\circ}$ at zero pressure. This reduction of the moiré pattern length for larger twist angle implies increase of the effective Coulomb interaction scale per moiré cell by about $50 \%$ and enhance roughly by a factor of $\sim 2$ the elastic energy that resists the commensuration strains due to the moiré pattern. Our results suggest that application of pressure on twisted bilayer graphene nanodevices through a hydraulic press will notably facilitate the device preparation efforts required for exploring the ordered phases near magic angle flatbands.

PACS numbers: 73.22.Pr, 31.15.aq
\end{abstract}

\section{INTRODUCTION}

The studies on the electronic properties of twisted bilayer graphene (tBLG) has received recently a renewed boost of interest thanks to groundbreaking discoveries of flatband superconductivity [1] and Mott gaps [2, 3] for certain magic angles close to $\sim 1^{\circ}$ where a high density of states is generated near the charge neutral point. The possibility of tailoring narrow flatbands in systems with such remarkable simplicity in composition as graphene consisting of only carbon atoms makes it an attractive testbed for trying to understand its microscopic mechanisms of electron correlations and its coupling with lattice vibrations as fully as possible [6-10]. Other van der Waals systems including trilayer graphene on $\mathrm{hBN}$, transition metal dichalcogenide layers manifesting flatbands and topological superlattice bands have been proposed in recent literature [1116].

In twisted bilayer graphene the graphene layers' electronic structure can be considered effectively decoupled when the twist angles are large enough, typically above $\theta \gtrsim 10^{\circ}$ as the twisted multilayer graphene stacks grown on $\mathrm{SiC}$ substrates [4, 5]. The progressive increase in coupling is manifested in the gradual decrease of the Fermi velocity as the twist angle is reduced starting from the Fermi velocity of graphene for large twist angles [17-21]. It is only in the regime of small twist angles on the order of $\sim 1^{\circ}$ that the moiré patterns are long enough to reduce the Fermi velocity band dispersion to the point of flattening the bands almost completely due to non-perturbative coupling between the electronic states separated by short moiré reciprocal lattice G-vectors in momentum space. In this limit a series of magic twist angles of $\theta=1.05^{\circ}, 0.5^{\circ}, 0.35^{\circ}, 0.24^{\circ}$, and $0.2^{\circ}$ have been predicted numerically [20] that approximately follow the $\theta \sim(1 / n)^{\circ}$ sequence for $n=1,2, \ldots$, where the band slopes assume zero values at the $\tilde{\Gamma}$ point of the moiré Brilouin zone, and the bandwidths achieve a series of minima. One important hurdle for the realization of the flatbands in twisted bilayer graphene lies at the high sensitivity of electronic structure to twist near the magic angles because the bandwidths can undergo variations on the order of $\sim 10 \mathrm{meV}$ for small variations in twist angle $\Delta \theta \sim 0.1^{\circ}$ leading to drastic changes in the electronic properties of tBLG.

In this work we show that application of appropriate pressure to the system is an additional control knob that can control the flatband bandwidth of the system. Contrary to the twist angles whose values are determined once for all for each fabricated device, the vertical pressure is a continuously variable system parameter controllable with a hydraulic press [22, 23] that should be applicable also in twisted bilayer graphene devices. Our work presents a roadmap for tailoring flatbands in twisted bilayer graphene even when the twist angle control is not strictly precise. We will begin by briefly introducing the model Hamiltonian used in our calculations, then move on to discuss the bandwidth phase diagram of the system as a function of pressure and twist angle, and further discuss the effects of an interlayer potential difference in the electronic structure of the flatbands before closing the paper with the summary and discussions.

\section{MOIRÉ PATTERN MODEL HAMILTONIAN}

The Hamiltonian of twisted bilayer graphene at valley $K$ is based on the continuum model of the graphene layer Hamiltonian [20] perturbed by stacking-dependent interlayer tunneling and intralayer potential variations [21]

$$
h(\boldsymbol{\theta})=\left(v_{F} \hat{P}_{\theta} \mathbf{p}+\mathbf{A}(\mathbf{r})\right) \cdot \sigma_{x y}+V(\mathbf{r}) \mathbb{1}+\Delta(\mathbf{r}) \sigma_{z}
$$

where $\sigma_{x y}=\left(\sigma_{x}, \sigma_{y}\right)$ and $\sigma_{z}$ are the graphene sublattice pseudospin Pauli matrices, the momentum is defined in the $x y$ plane $\mathbf{p}=\left(p_{x}, p_{y}\right)$ and $\hat{P}_{\theta}$ introduces a phase shift in the offdiagonal term in the Dirac Hamiltonian to account for the rotation of the layers $e^{ \pm i \theta_{\mathbf{k}}} \rightarrow e^{ \pm i\left(\theta_{\mathbf{k}}-\theta\right)}$ where $\theta_{\mathbf{k}}$ is measured with respect to the $x$-axis and $\theta$ is the rotation of the graphene layer with respect to $x$. The graphene layers can be coupled through a stacking-dependent interlayer tunneling $T(\mathbf{r})$ [20] 
resulting in the Hamiltonian

$$
H_{\mathrm{tBLG}}=\left(\begin{array}{cc}
h(-\theta / 2) & T(\mathbf{r}) \\
T^{\dagger}(\mathbf{r}) & h(\boldsymbol{\theta} / 2)
\end{array}\right) .
$$

The symmetric and opposite rotations of the top and bottom graphene layers allows to preserve the moiré Brillouin zone (mBZ) orientation and therefore of the stacking dependent moiré patterns $V(\mathbf{r}), \Delta(\mathbf{r}), \mathbf{A}(\mathbf{r})$ for intralayer potential variations, local mass term and virtual strains due to pseudomagnetic field vector potentials, in addition to the spatially varying interlayer tunneling $T(\mathbf{r})$ for every position $\mathbf{r}$. Using the first harmonic approximation we have

$$
M(\mathbf{r})=\sum_{m=1}^{6} e^{i \mathbf{G}_{m} \mathbf{r}} M_{m}
$$

where the moiré patterns $M(\mathbf{r})$ can be modeled through its Fourier coefficients $M_{m}$. More explicitly, for a triangular lattice we can write the scalar and vector moiré patterns as [21, 24]

$$
\begin{aligned}
& V(\mathbf{r})=2 C_{V} \operatorname{Re}\left[e^{i \phi_{V}} f(\mathbf{r})\right], \Delta(\mathbf{r})=2 C_{\Delta} \operatorname{Re}\left[e^{i \phi_{\Delta}} f(\mathbf{r})\right] \\
& \mathbf{A}(\mathbf{r})=2 C_{A B} \hat{z} \times \nabla \operatorname{Re}\left[e^{i \phi_{x y}} f(\mathbf{r})\right]
\end{aligned}
$$

where we have used the auxiliary function $f(\mathbf{r})=$ $\sum_{m=1}^{6} e^{i \mathbf{G}_{m} \cdot \mathbf{r}}\left(1+(-1)^{m}\right) / 2$, where the six first shell $\mathrm{mBZ}$ recirpocal lattice vectors are $\mathbf{G}_{m}=\hat{R}_{2 \pi(m-1) / 3} \mathbf{G}_{1}$ for $m$ indices running from 1 to 6 are generated through successive rotation by $2 \pi / 3$ of the vector $\mathbf{G}_{1} \simeq(0,4 \pi \theta / \sqrt{3} a)$, where $a=2.46 \AA$ is the lattice constant of graphene. The moiré pattern Hamiltonian parameters obtained from ab initio calculations in sublattice representation for rigid bilayer graphene are [21] $C_{A A}=$ $C_{B^{\prime} B^{\prime}}=1.10 \mathrm{meV}, \varphi_{A A}=\varphi_{B^{\prime} B^{\prime}}=82.54^{\circ}, C_{B B}=C_{A^{\prime} A^{\prime}}=C_{A A}$, $\varphi_{B B}=\varphi_{A^{\prime} A^{\prime}}=-\varphi_{A A}, C_{A B}=2.235 \mathrm{meV}, \varphi_{A B}=0^{\circ}$, and with LDA out of plane relaxation we have $C_{A A}=2.3 \mathrm{meV}, \varphi_{A A}=$ $27.5^{\circ}, C_{B B}=C_{A A}, \varphi_{B B}=-\varphi_{A A}, C_{A B}=2.08 \mathrm{meV}, \varphi_{A B}=0^{\circ}$, where we use the notation $\phi_{x y}=\pi / 6-\varphi_{A B}$. In our modeling of the rigid twisted bilayers the intralayer moiré patterns have a small effect in the electronic structure and they can be neglected, whereas the relaxed moiré pattern parameters lead to particle-hole symmetry breaking as we will show later on. The momentum conservation condition in twisted bilayer graphene

$$
\mathbf{k}^{\prime}=\mathbf{k}+\mathbf{G}
$$

implies that a Bloch state with momentum $\mathbf{k}$ from one layer scatters to $\mathbf{k}^{\prime}$ at the other layer through a moiré reciprocal lattice vector $\mathbf{G}[21]$. In the small angle approximation we have $\mathbf{G} \simeq-\theta \hat{z} \times \mathbf{g}$ where the reciprocal lattice vector of graphene is represented through $\mathbf{g}$. If we consider the $\mathbf{q}=\mathbf{k}-\mathbf{K}$ and $\mathbf{q}^{\prime}=\mathbf{k}^{\prime}-\mathbf{K}^{\prime}$ momenta measured respect to the Dirac points of each layer relatively displaced by $\Delta \mathbf{K}=\mathbf{K}^{\prime}-\mathbf{K}=2 K \sin (\theta / 2)$ we have the relationship $\mathbf{q}^{\prime}=\mathbf{q}+\mathbf{K}-\mathbf{K}^{\prime}+\mathbf{G}=\mathbf{q}+\mathbf{Q}$, where the three $\mathbf{Q}_{j}$ vectors given by $\mathbf{Q}_{0}=K \boldsymbol{\theta}(0,-1)$ and $\mathbf{Q}_{ \pm}=$ $K \theta( \pm \sqrt{3} / 2,1 / 2)$ in the small angle approximation to represent the interlayer coupling Hamiltonian

$$
T(\mathbf{r})=\sum_{j} e^{-i \mathbf{Q}_{j} \mathbf{r}} T_{s, s^{\prime}}^{j},
$$

where the interlayer coupling matrices are given by

$$
T^{0}=\omega\left(\begin{array}{ll}
1 & 1 \\
1 & 1
\end{array}\right), T^{ \pm}=\omega\left(\begin{array}{cc}
1 & e^{\mp i 2 \pi / 3} \\
e^{ \pm i 2 \pi / 3} & 1
\end{array}\right)
$$

We note that these $T^{j}$ matrices result when the twist is applied to a bilayer with $\tau=(0,0)$ initial AA stacking configuration and differ by an additional phase of $e^{-i \mathbf{G}_{j} \tau}$ with respect to the $\mathrm{AB}$ stacking case where $\tau=(0, a / \sqrt{3})$. The smooth variation of $T(\mathbf{r})$ in Eq. (7) can be traced back to the relatively larger interlayer distance of $c \sim 3.35 \AA$ when compared to inter-carbon distances of $a_{\mathrm{CC}} \sim 1.42 \AA$ [20] and effectively implies that the bilayer graphene interlayer coupling strength can be described by a single parameter $\omega=t_{1} / 3 \sim 0.113 \mathrm{eV}$ when $c=3.35 \AA$, and somewhat weaker $\omega \sim 0.1 \mathrm{eV}$ when out of plane LDA relaxations are allowed between the layers [21]. We note that $\omega$ is proportional to the interlayer coupling term $t_{1} \sim 3 \omega$ of commensurate bilayer graphene evaluated at the Dirac point when only the three $\mathbf{G}$-vector contributions of interlayer coupling nearest to the Dirac point $K$ are considered. The interlayer coupling can in principle be modeled more accurately by including the effects of the Fourier components for larger $\mathbf{G}$-vectors in momentum space that become more relevant in the presence of out of plane corrugations and inplane strains [21, 25]. Given the high accuracy of the single parameter interlayer coupling model for describing the electronic properties of rigid twisted layers, we will focus on the role of $\omega$ in our precisely defined continuum model and defer the discussions about the moiré strains and larger momenta Fourier components for future work.

\section{PRESSURE DEPENDENT FLATBAND BANDWIDTHS}

In the following we obtain the phase diagram map of the bandwidth as a function of pressure to show how increase in pressure by a few GPa can trigger the appearance of flatbands in tBLG for twist angles that are larger by a fraction of a degree than the magic angles at zero pressure. We have calculated the phase diagram map of the moiré bands bandwidth of twisted bilayer graphene as a function of the three main parameters in the model Hamiltonian, namely the Fermi velocity of each graphene layer $v_{\mathrm{F}}$, the twist angle $\theta$, and the pressure dependent interlayer coupling strength $\omega$. The Fermi velocity $v_{\mathrm{F}}$ can change with the dielectric environment due to many-body effects but otherwise can be assumed to be constant. The twist angle $\theta$ can be controlled at will during device fabrication but is difficult to modify afterwards in a controlled manner, whereas the pressure that controls the interlayer coupling $\omega$ can in principle be varied continuously in a hydraulic press up to values of $2.5 \mathrm{GPa}$ [22]. Our main results are summarized in Fig. 1 where we represent the flatband bandwidth colormap obtained as a function of interlayer coupling parameter $\omega$ and twist angle $\theta$. Note that the bandwidth for the low energy conduction and valence bands are electron-hole symmetric in the absence of intralayer moiré pattern terms in the 


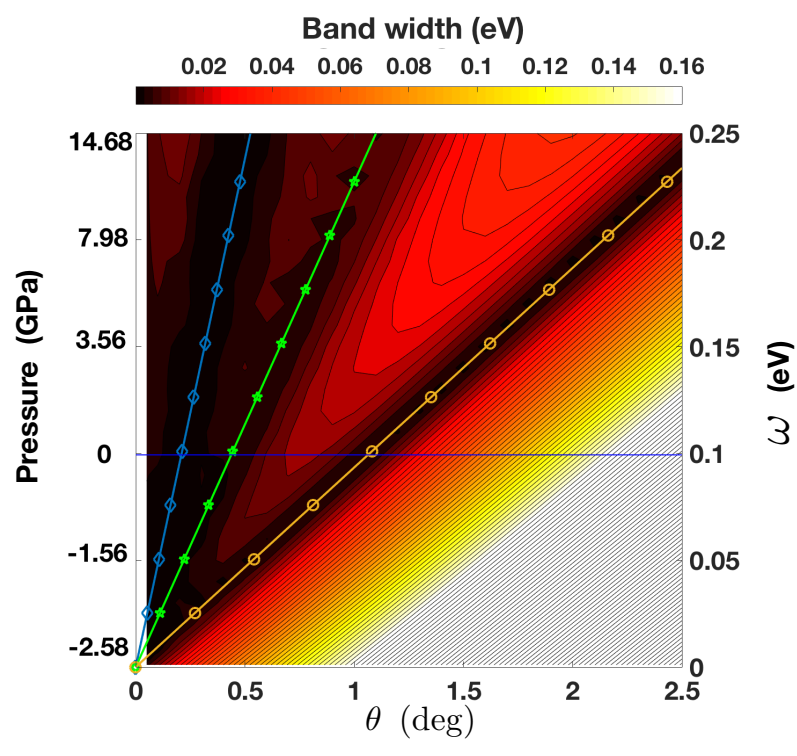

FIG. 1. (Color online) Colormap of the flatband bandwidth as a function of twist angle $\theta$ and interlayer tunneling strength $\omega$. We identify numerically straight lines in the parameter space given by Eq. (9), where we represent in orange, green and blue the regions in the phase diagram corresponding to the first, second and third magic angles for decreasing twist angles for which bandwidth minima are achieved. We have verified that the straight lines in $\theta$ and $\omega$ fit accurately the bandwidth minima for a variety of of $v_{\mathrm{F}}=3\left|t_{0}\right| a / 2 \hbar$. The interlayer tunneling value of $\omega \sim 0.11 \mathrm{eV}$ corresponds to rigid graphene at fixed $c=3.35 \AA$ interlayer spacing, while out of plane relaxations within LDA reduces the effective tunneling to $\omega \sim 0.1 \mathrm{eV}$ (blue horizontal line) when no external pressure is applied.

Hamiltonian. From the analysis of the numerically calculated bandwidth phase diagrams for different Fermi velocities we find a fitting formula for the three visible flatband magic angle lines

$$
\theta_{n}^{\circ}=C_{n} \frac{\omega}{\left|t_{0}\right|} \quad(\mathrm{deg})
$$

where $C_{1}=27.8, C_{2}=11.5$, and $C_{3}=5.46$, that lead to $\theta_{1}=1.07^{\circ}, \theta_{2}=0.44^{\circ}$ and $\theta_{3}=0.21^{\circ}$ with $t_{0}=-2.6 \mathrm{eV}$ and $\omega=0.1 \mathrm{eV}$. These results are in fair agreement with the numerical magic angles in Ref. [20] that approximately follow the $\theta \sim(1 / n)^{\circ}$ sequence for $t_{0}=-2.7 \mathrm{eV}$ and $\omega=$ $0.11 \mathrm{eV}$ Hamiltonian parameters, except for the angles of $\theta=0.35^{\circ}, 0.24^{\circ}$ which are not clearly resolved in our bandwidth phase diagram. Our flatband magic angles are linearly proportional to the interlayer coupling $\omega$, and are inversely proportional to the Fermi velocity $v_{\mathrm{F}}=\sqrt{3}\left|t_{0}\right| a / 2 \hbar$. We note that in our case the magic angles were obtained from bandwidth minima in the $\omega$ and $\theta$ parameter space, while previous calculations identified the magic twist angles from the zero slope in the band dispersion at the $\tilde{\Gamma}$-point in the mBZ. One practical implication of our findings is that it should be possible to access the flatbands by increasing $\omega$ with pressure by a few GPa when the twist angles in bilayer graphene are a fraction of a degree larger than the zero pressure magic angles.

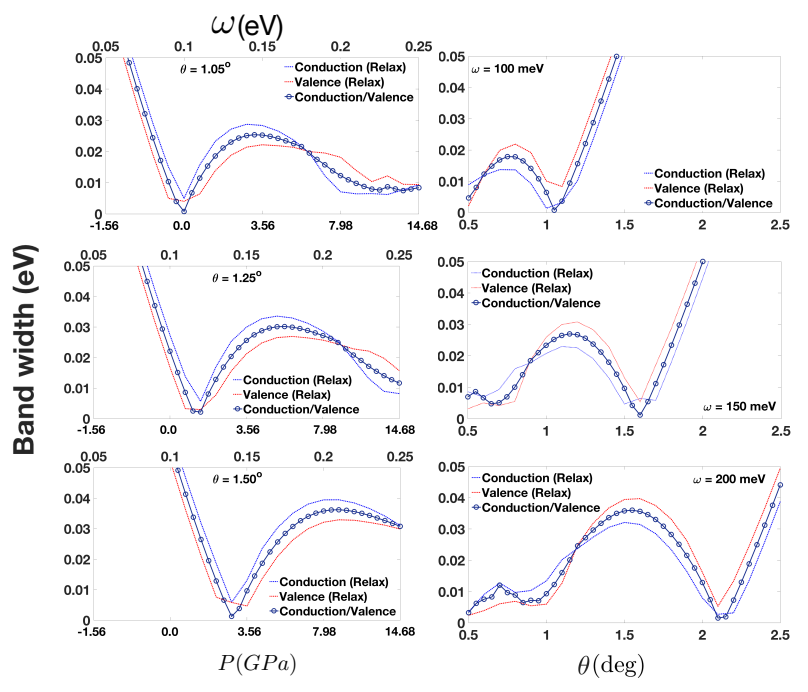

FIG. 2. (Color online) Flatband bandwidth evolution as a function of pressure and twist angle for the rigid continuum model with negligible intralayer moire patterns and the out of plane relaxed model that introduces a small particle hole asymmetry in the low energy bands. Left Panel: Flatband bandwidth as a function of $\omega$ (or external pressure $P$ ), for different values of the twist angle $\theta^{\circ}=1^{\circ}, 1.25^{\circ}, 1.5^{\circ}$. We observe a progressive reduction in the bandwidth as pressure is increased from left to right until it arrives to a minimum value. Right Panel: Flatband bandwidth as a function of twist angle $\theta$ for different constant values of interlayer coupling $\omega=100,150,200 \mathrm{meV}$. We observe that the bandwidth has a non-monotonic dependence with respect to the twist angle with almost vanishing bandwidth for the first magic angle but maintaining a finite value for the second magic angle.

The Fermi velocity that we use as one of the free parameters in our model is an intrinsic property of graphene that can be enhanced by Coulomb interactions and is therefore subject to specific environment and device quality. Strictly speaking, a logarithmic divergence is expected for the Fermi velocity at close proximity of the Dirac point due to the long rangedness of the Coulomb tail which introduces $k$-dependent dispersion slope changes [26-28]. Yet a constant enhanced Fermi velocity often gives an excellent fit to experimental data, with the lower $v_{F} \sim 1 \times 10^{6}$ or $\sim 1.05 \times 10^{6}$ fitting well experiments of graphene on $\mathrm{SiC}$ or $\mathrm{SiO}_{2}$ substrates [4, 29] and CVD grown twisted bilayer graphene [30], while higher $v_{F} \sim 1.1 \times 10^{6} \mathrm{~m} / \mathrm{s}$ are better for fitting the experimental data in high quality graphene devices with hexagonal boron nitride barrier materials [31]. In this work, we used ab initio LDA calculation values of $t_{0}=-2.6 \mathrm{eV}$ for the intralayer hopping term [32] that is in the lower end of the spectrum with $v_{\mathrm{F}} \sim 0.84 \times 10^{6}$, and close to $t_{0} \sim-2.7 \mathrm{eV}$ used in Ref. [20]. Our tight-binding Fermi velocity choice is more appropriate for band theories that intend to introduce the many-body corrections explicitly on top of the non-interacting model.

The twist angles range examined in the phase diagram lie between $0.05^{\circ}$ and $2.5^{\circ}$. Convergence of the eigenvalues 

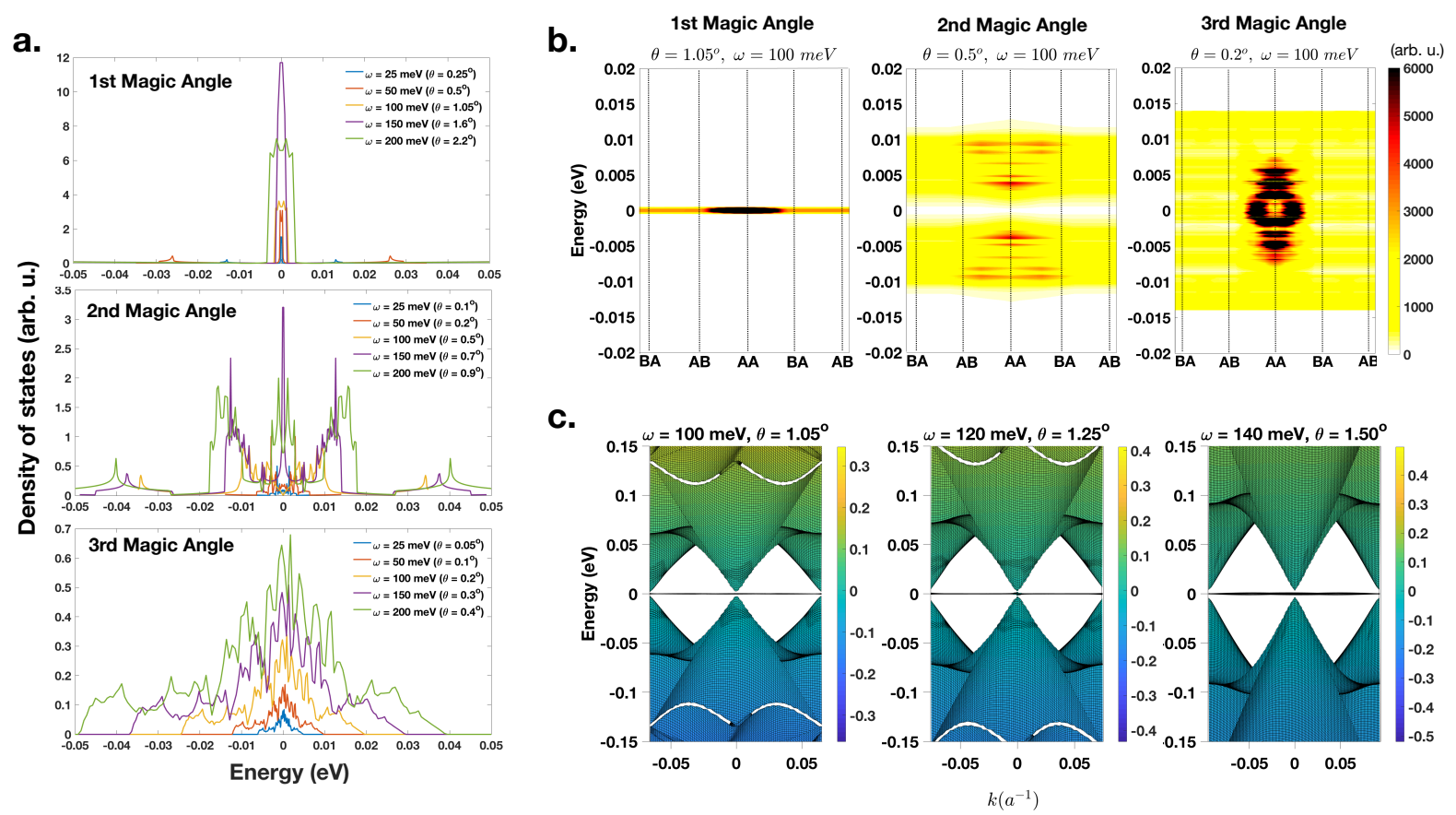

FIG. 3. (Color online) a. Density of states (DOS) of the flatbands corresponding to the first (top panel), second (middle panel) and third (bottom panel) magic angles for different values of interlayer tunneling $\omega$. We observe a progressive increase in the DOS maxima and width variations for larger twist angle flat bands due to the increase in the moiré Brillouin zone area. b. Local density of states evaluated for the first, second and third magic angles along lines connecting different local stacking configurations. While the wave functions still localize at AA stacking regions the flatbands bandwidth widen for higher order magic angles. c. Band structure plots for different interlayer coupling strength and progressive increase in the magnitude of the first magic angles due to enhancement of interlayer tunneling achievable applying pressure.

and eigenvectors of our continuum model can be expected when truncation of the moiré reciprocal lattice vector is of the order of $k \sim 2 \omega /\left(\sqrt{3} a\left|t_{0}\right|\right)$ [20], requiring a larger cutoff when $\omega$ is larger. We have used a cutoff in momentum space for a radius of about six moiré reciprocal lattice vectors $6 G_{1}=24 \pi \theta /(\sqrt{3} a)$ using fixed Hamiltonian matrix sizes of $676 \times 676$ to obtain the phase diagram in Fig. 1. which should be valid for sufficiently large $\theta \gtrsim \omega /\left(12 \pi\left|t_{0}\right|\right)$ or in the limit of small $\omega$. Our model assumes rigid in-plane lattices although for systems with small twist angles $\theta \lesssim 0.5^{\circ}$ one expects structural instabilities associated with the commensuration moiré strains due to the reduction of the elastic energies that scale with the twist angle as $\propto \theta^{2}$ [25].

Pressure can be varied in a continuous manner even after device fabrication to modify the magnitude of interlayer tunneling. Recent experimental progress that make use of a hydraulic press allowed to achieve continuously variable pressures of up to $2.5 \mathrm{GPa}$ for $\mathrm{hBN}$ encapsulated graphene devices while values as large as $5 \mathrm{GPa}$ might be achievable by optimizing the design of the press, and even larger pressure would be achievable with diamond anvil cells. These values are still safely below pressures where structural phase transitions from graphite to diamond can start taking place [33, 34]. The relationship between the interlayer coupling parameter $\omega$ and pressure $P$ is obtained by combining Fourier transformed interlayer hopping data between maximally local- ized Wannier functions obtained from LDA ab initio calculations evaluated at three fixed interlayer separation distances of $c=3.35,3.2,3.1 \AA$ for different interlayer stacking. The pressures for different interlayer distances at $A B$ and $B A$ stacking configurations within the LDA are given by $P=0,2.01$ and $3.45 \mathrm{GPa}$, and larger values for AA stacking of $P=2.09,4.80,7.73 \mathrm{GPa}$ within LDA consistent with the calculations in Ref. [35], which can be fitted with the Murnaghan equation of state $P(V)=\left(K_{0} / K_{0}^{\prime}\right)\left[\left(V / V_{0}\right)^{-K_{0}^{\prime}}-1\right]$ [36] or the third order Birch-Murnaghan equation of state [37] using the parameters listed in Table [1 By assuming approximately equal distribution of $\mathrm{AB}, \mathrm{BA}$ and $\mathrm{AA}$ stacking areas, and averaging the values of interlayer tunneling at the Dirac point for different stacking configurations we obtain a polynomial fit for the relationship between pressure and averaged interlayer tunneling $\omega$ through

$$
P=A \omega^{2}+B \omega+C,
$$

whose numerical parameters are $A=455.5 \mathrm{GPa} /(\mathrm{eV})^{2}, B=$ $-71.05 \mathrm{GPa} / \mathrm{eV}, C=3.281 \mathrm{GPa}$ and $\omega_{0}=0.098 \mathrm{eV}$ is the tunneling for $P=0$. Because the tunneling is weaker for larger interlayer distances our $\omega_{0}$ consistent with relaxed LDA results is smaller than $\omega=0.113 \mathrm{eV}$ obtained from the average of rigid graphene bilayers separated at fixed $c=3.35 \AA$ [21]. For sake of definiteness we will use for our zero pressure continuum model Hamiltonian the parameters $t_{0}=-2.6 \mathrm{eV}$ and 


\begin{tabular}{|c|cc|cc|cc|}
\hline & \multicolumn{3}{|c|}{ AA } & \multicolumn{2}{c|}{ AB } & \multicolumn{2}{c|}{ BA } \\
\hline & LDA & RPA & LDA & RPA & LDA & RPA \\
\hline$K_{0}$ & 322 & 353 & 308 & 358 & 308 & 358 \\
$K_{0}^{\prime}$ & 10.88 & 12.59 & 12.51 & 12.02 & 12.51 & 12.02 \\
$B_{0}$ & 239 & 331 & 302 & 354 & 302 & 354 \\
$B_{0}^{\prime}$ & 14.27 & 15.00 & 14.25 & 13.48 & 14.25 & 13.48 \\
\hline
\end{tabular}

TABLE I. Bulk moduli $K_{0}$ and $K_{0}^{\prime}$ for Murnaghan and $B_{0}$ and $B_{0}^{\prime}$ in kbar units for the third-order Birch-Murnaghan equations of state consistent with the interlayer potentials obtaiend in Ref. [35] obtained from ab initio calculations for different local stacking configurations.

$\omega=0.1 \mathrm{eV}$ that leads to electronic structure results closely similar to those obtained in Ref. [20]. The weaker interlayer tunneling regime $\omega<0.1 \mathrm{eV}$ in the phase diagram might be achievable in systems whose average interlayer distances can be reduced either through intercalation of ions or addition of a barrier hBN. The use of an intercalation hBN spacer layer between the top and bottom graphene layers to reduce interlayer tunneling can prevent the structural instabilities for small twist angles that are present when both graphene layers are in direct contact.

\section{DENSITY OF STATES}

The sharp increase in the density of states (DOS) and its width at the flatbands relative to Coulomb interaction strength determines how prone the system is towards the development of broken symmetry phases. Here we analyze the impact that the interlayer coupling strength $\omega$ has in the density of states (DOS) when its value is increased along the flatband line equation given in Eq. (9) for a few select twist angles and interlayer tunneling $\omega$. The increase of the interlayer tunneling parameter $\omega$ expands the energy range around which a given Bloch state in one layer can scatter to the other layer and affects the eigenvectors associated with the flatbands. An immediate effect of having larger magic twist angles is that we can expect an enhancement of the flatband DOS due to the increase of $\mathrm{mBZ}$ area proportionally to $\theta^{2}$, making more states accessible in momentum space, because more electrons per unit area are required to fill the same number of the moire flatbands. The reduction in the moire pattern length implies a relative enhancement of the effective Coulomb interaction scale $U_{\text {eff }}=e^{2} /\left(4 \pi \varepsilon_{0} \varepsilon_{r} \ell_{M}\right) \sim e^{2} \theta /\left(4 \pi \varepsilon_{0} \varepsilon_{r} a\right)$ where $\ell_{M} \sim a / \theta$, and therefore an increase from $\theta \sim 1^{\circ}$ to $1.5^{\circ}$ leads to already a $50 \%$ increase. We show in Fig. 3 the density of states and the local density of states associated with the charge neutrality flatbands for different values of twist angle $\theta$ and interlayer tunneling $\omega$ where we can observe a steady increase in the flatband DOS either for its peak height and width for progressively larger twist angle sizes. The broadening of the flatbands for smaller magic angles widens of the energy range around which the LDOS concentration takes place, reflecting the increase of interlayer coherence.

\section{SUMMARY AND DISCUSSIONS}

In summary we have investigated the phase diagram map of the low energy bandwidth evolution in twisted bilayer graphene as a function of parameters in the continuum model Hamiltonian including the Fermi velocity $v_{\mathrm{F}}$, the twist angle $\theta$ and the interlayer tunneling parameter $\omega$ in search of the phase space where we can achieve bandwidth minima, in particular when the interlayer tunneling is enhanced by means of external pressure. The flatbands for the continuum Hamiltonian can be summarized in a single line equation relating the minimum bandwidth magic twist angle with interlayer tunneling, and is inversely proportional to the Fermi velocity or intralayer hopping. Our calculations indicate that by applying pressures on the order of $\sim 2 \mathrm{GPa}$ achieved in recent experiments through a hydraulic press [22] it should be possible to access the first magic angle around $\theta \sim 1.5^{\circ}$ which is considerably greater than $\sim 1^{\circ}$ and therefore should have considerably greater structural stability that can be altered by the moiré strains and have enhanced effective Coulomb interaction energy scales $U_{\text {eff }} \propto \ell_{M}^{-1} \propto \theta$ thanks to the reduction in the moiré pattern size. Hence, we can envision that application of pressure in twisted bilayer graphene nanodevices to achieve larger magic angles should considerably facilitate access to flatbands and electron-electron interaction driven ordered phases.

\section{ACKNOWLEDGEMENT}

Support from the Korean National Research Foundation is acknowledged for B.L.C. through NRF2017R1D1A1B03035932, for N. L. through NRF2018R1C1B6004437 and for J.J. through NRF2016R1A2B4010105. N.L. and J.J. also acknowledge support by the Korea Research Fellowship Program through the National Research Foundation of Korea (NRF) funded by the Ministry of Science and ICT(KRF-2016H1D3A1023826). A.H.M. acknowledges financial support by the Army Research Office (ARO) under contract W911NF-15-10561:P00001, and by the Welch Foundation under grant TBF1473.

[1] Y. Cao, V. Fatemi, S. Fang, K. Watanabe, T. Taniguchi, E. Kaxiras, and P. Jarillo-Herrero, Nature 556, 43-50 (2018).

[2] Y. Cao, V. Fatemi, A. Demir, S. Fang, S. L. Tomarken, J. Y. Luo, J. D. Sanchez-Yamagishi, K. Watanabe, T. Taniguchi, E. Kaxiras, R. C. Ashoori, and P. Jarillo-Herrero, Nature 556, 8084 (2018).

[3] K. Kim, A. DaSilva, S. Huang, B. Fallahazad, S. Larentis, T. Taniguchi, K. Watanabe, B. J. LeRoy, A. H. MacDonald, and E. Tutuc, PNAS 114 (13), 3364-3369, (2017).

[4] C. Berger et al., Science 312, 1191 (2006).

[5] S.Y. Zhou, G.-H. Gweon, A.V. Fedorov, P.N. First, W.A. de Heer, D.-H. Lee, F. Guinea, A.H. Castro Neto, A. Lanzara, Na- 
ture Materials 6, 770-775 (2007).

[6] N. F. Q. Yuan, L. Fu, Phys. Rev. B 98, 045103 (2018).

[7] F. Wu, A. H. MacDonald, I. Martin, arXiv:1805.08735

[8] C. Li, M. Franz, arXiv:1806.06092

[9] B. Lian, Z. Wang, B. Andrei Bernevig arXiv:1807.04382

[10] D. M. Kennes, J. Lischner, and C. Karrasch, arXiv:1805.06310

[11] F. Wu, T. Lovorn, E. Tutuc, A. H. MacDonald, arXiv:1804.03151 (2018).

[12] Y. Pan, S. Folsch, Y. Nie, D. Waters, Y.-C. Lin, B. Jariwala, K. Zhang, K. Cho, J. A. Robinson, and R. M. Feenstra, Nano Lett. 18, 1849-1855 (2018).

[13] S. Carr, D. Massatt, M. Luskin, E. Kaxiras, arXiv:1803.01242 (2018).

[14] G. Chen, L. Jiang, S. Wu, B. Lv, H. Li, K. Watanabe, T. Taniguchi, Z. Shi, Y. Zhang, F. Wang, arXiv:1803.01985 (2018).

[15] B. L. Chittari, G. Chen, Y. Zhang, F. Wang, J. Jung, arXiv:1806.00462 (2018).

[16] Y.-H. Zhang, D. Mao, Y. Cao, P. Jarillo-Herrero, and T. Senthil, arXiv:1805.08232

[17] J. M. B. Lopes dos Santos, N. M. R. Peres, A. H. Castro Neto, Phys. Rev Lett. 99256802 (2007).

[18] Z. F. Wang, Feng Liu, and M. Y. Chou, Nano Lett., 12 (7), 3833-3838 (2012).

[19] P. San-Jose, E. Prada, Phys. Rev. B 88, 121408(R) (2013).

[20] R. Bistritzer and A. H. MacDonald, PNAS 108 (30), 1223312237 (2011).

[21] J. Jung, A. Raoux, Z. Qiao, and A. H. MacDonald, Phys. Rev. B 89, 205414 (2014).

[22] M. Yankowitz, J. Jung, E. Laksono, N. Leconte, B. L. Chittari, K. Watanabe, T. Taniguchi, S. Adam, D. Graf, and C. R. Dean, Nature 557, 404-408 (2018).
[23] S. Carr, S. Fang, P. Jarillo-Herrero, E. Kaxiras, 1806.05078 (2018).

[24] J. Jung, E. Laksono, A. M. DaSilva, A. H. MacDonald, M. Mucha-Kruczyski, and S. Adam, Phys. Rev. B 96, 085442 (2017).

[25] J. Jung, A. M. DaSilva, A. H. MacDonald, and S. Adam, Nat. Comm. 6, 6308 (2015).

[26] J. González, F. Guinea, and M. A. H. Vozmediano, Phys. Rev. B 59, R2474(R) (1999).

[27] J. Jung, A. H. MacDonald, Phys. Rev. B 84, 085446 (2011).

[28] Y. Barlas, T. Pereg-Barnea, M. Polini, R. Asgari, and A. H. MacDonald, Phys. Rev. Lett. 98, 236601 (2007).

[29] C. Faugeras, M. Amado, P. Kossacki, M. Orlita, M. Kühne, A. A. L. Nicolet, Y. I. Latyshev, and M. Potemski, Phys. Rev. Lett. 107, 036807 (2011).

[30] D. Wong, Y. Wang, J. Jung, S. Pezzini, A. M. DaSilva, H.-Z. Tsai, H. S. Jung, R. Khajeh, Y. Kim, J. Lee, S. Kahn, S. Tollabimazraehno, H. Rasool, K. Watanabe, T. Taniguchi, A. Zettl, S. Adam, A. H. MacDonald, and M. F. Crommie, Phys. Rev. B 92, 155409 (2015).

[31] K. Lee, J. Jung, B. Fallahazad and E. Tutuc, 2D Mater. 4, 035018 (2017).

[32] J. Jung and A. H. MacDonald, Phys. Rev. B 87, 195450 (2013).

[33] Yagi T, Utsumi W, Yamakata Ma, Kikegawa T, Shimomura O, Phys Rev B, 46, 6031-6039 (1992).

[34] Hanfland M, Syassen K, Sonnenschein R, Phys Rev B, 40, 1951-1954 (1989).

[35] N. Leconte, J. Jung, S. Lebegue, and T. Gould, Phys. Rev. B 96, 195431 (2017).

[36] F.D. Murnaghan, PNAS 30:244-247 (1944).

[37] F. Birch, Phys. Rev. 71, 809 (1947). 